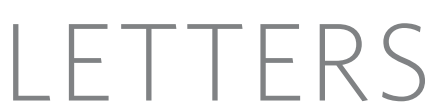

\title{
Mutation of FIG4 causes neurodegeneration in the pale tremor mouse and patients with CMT4J
}

\author{
Clement Y. Chow ${ }^{1}$, Yanling Zhang ${ }^{2}$, James J. Dowling ${ }^{4}$, Natsuko Jin ${ }^{2}$, Maja Adamska ${ }^{1}$, Kensuke Shiga $^{5}$, Kinga Szigeti ${ }^{5,7}$, \\ Michael E. Shy ${ }^{9}$, Jun Li ${ }^{9,10}$, Xuebao Zhang ${ }^{9}$, James R. Lupski ${ }^{5,6,8}$, Lois S. Weisman ${ }^{2,3}$ \& Miriam H. Meisler ${ }^{1}$
}

Membrane-bound phosphoinositides are signalling molecules that have a key role in vesicle trafficking in eukaryotic cells ${ }^{1}$. Proteins that bind specific phosphoinositides mediate interactions between membrane-bounded compartments whose identity is partially encoded by cytoplasmic phospholipid tags. Little is known about the localization and regulation of mammalian phosphatidylinositol-3,5-bisphosphate (PtdIns $\left.(3,5) \mathrm{P}_{2}\right)$, a phospholipid present in small quantities that regulates membrane trafficking in the endosome-lysosome axis in yeast ${ }^{2}$. Here we describe a multi-organ disorder with neuronal degeneration in the central nervous system, peripheral neuronopathy and diluted pigmentation in the 'pale tremor' mouse. Positional cloning identified insertion of $E T n 2 \beta$ (early transposon $2 \beta)^{3}$ into intron 18 of Fig4 (A530089I17Rik), the homologue of a yeast SAC (suppressor of actin) domain PtdIns (3,5) $\mathrm{P}_{2}$ 5-phosphatase located in the vacuolar membrane. The abnormal concentration of $\operatorname{PtdIns}(3,5) \mathrm{P}_{2}$ in cultured fibroblasts from pale tremor mice demonstrates the conserved biochemical function of mammalian Fig4. The cytoplasm of fibroblasts from pale tremor mice is filled with large vacuoles that are immunoreactive for LAMP-2 (lysosomal-associated membrane protein 2), consistent with dysfunction of the late endosome-lysosome axis. Neonatal neurodegeneration in sensory and autonomic ganglia is followed by loss of neurons from layers four and five of the cortex, deep cerebellar nuclei and other localized brain regions. The sciatic nerve exhibits reduced numbers of largediameter myelinated axons, slowed nerve conduction velocity and reduced amplitude of compound muscle action potentials. We identified pathogenic mutations of human FIG4 (KIAA0274) on chromosome $6 \mathrm{q} 21$ in four unrelated patients with hereditary motor and sensory neuropathy. This novel form of autosomal recessive Charcot-Marie-Tooth disorder is designated CMT4J.

Mutant mice with severe tremor, abnormal gait and diluted pigmentation were identified in our mouse colony on a mixed inbred strain background. One breeding pair generated 8/30 affected progeny, consistent with inheritance of an autosomal recessive mutation designated 'pale tremor'. At postnatal day three (P3), affected homozygotes have diluted pigmentation and reduced size (Fig. 1a). Intentional tremor develops during the second week after birth, and abnormal limb postures are evident by the third week (Fig. 1b). The impaired motor coordination, muscle weakness and 'swimming' gait of pale tremor mice are demonstrated in the Supplementary Movie. There is progressive loss of mobility, reduction in body weight and juvenile lethality (Fig. 1c).

To genetically map the pale tremor gene, a cross was carried out with strain CAST/Ei. The recovery of affected $\mathrm{F}_{2}$ offspring was $9 \%$ (50/532), indicative of prenatal loss on this genetic background. We genotyped $532 \mathrm{~F}_{2}$ animals with microsatellite and single nucleotide polymorphism markers to map the pale tremor gene to a 2-megabase interval of mouse chromosome 10 between D10Umi13 and D10Mit184 (Supplementary Fig. 1a). There are 21 annotated genes in the non-recombinant interval (http://www.ensembl.org; mouse build 35); these were tested as candidates by sequencing reverse transcription polymerase chain reaction (RT-PCR) products from brain RNA. The A530089I17Rik transcript amplified from pale tremor RNA lacks exons 19 to 23 from the $3^{\prime}$ end of the gene (Fig. 1d). Hybridization of a polyA ${ }^{+}$northern blot with a complementary DNA probe containing exons 8 to 15 identified a wild-type transcript of 3.3 kilobases $(\mathrm{kb})$ that is not present in messenger RNA from pale tremor mice (Fig. 1e). No abnormal transcripts were detected in the mutant, even when the exposure time was increased from 3 to $63 \mathrm{~h}$ (not shown). We were able to amplify exons 19 to 23 from genomic DNA, eliminating the possibility of a genomic deletion (Supplementary Fig. 2a).

To identify the genomic mutation, we examined the structure of intron 18 using PCR. A wild-type product of $0.65 \mathrm{~kb}$ and a mutant product of $6.2 \mathrm{~kb}$ were amplified from the $3^{\prime}$ end of intron 18 (Fig. 1f). The sequence of the mutant product (GenBank DQ813648) contains a 5,547 base pair (bp) insert with $99 \%$ sequence identity to mouse retrotransposon ETn2 $\beta$ (GenBank Y17106). The transposon is inserted 384 bp upstream of exon 19, in the same orientation as the gene (Fig. $1 \mathrm{~h}$ ), and is flanked by a duplication of the hexanucleotide CCCCTG, characteristic of $E T n 2 \beta$ insertions ${ }^{3}$. The mutant allele can be detected by PCR with a primer in the long terminal repeat (Fig. 1g). The background strains do not contain the ETn $2 \beta$ element (data not shown), indicating that mutation of the pale tremor gene is a result of transposon insertion. The data are consistent with abnormal splicing from exon 18 of Fig4 to one of the cryptic splice acceptor sites in the ETn $2 \beta$ element $^{3}$, generating a transcript of very low abundance that is detected by RT-PCR but is below the sensitivity of the polyA ${ }^{+}$northern blot.

RT-PCR of tissues from wild-type mice demonstrated widespread expression of Fig4 (Supplementary Fig. 2b), consistent with public expressed-sequence-tag and microarray databases. In situ hybridization data demonstrate distribution of the transcript throughout the brain (http://www.brainatlas.org/aba/ and unpublished observations, C.Y.C. and M.H.M.). The human orthologue, KIAA0274, is located on human chromosome 6q21. The mutated protein is most closely related to the yeast Saccharomyces cerevisiae SAC-domain phosphatase Fig4, with overall amino acid sequence identity of $35 \%$ and similarity of $66 \%$. The SAC domain with the active site motif $\mathrm{CX}_{5} \mathrm{R}(\mathrm{S} / \mathrm{T})$ is characteristic of phosphatases with specificity for phosphoprotein or phospholipid substrates ${ }^{4}$, and exhibits $44 \%$ sequence identity (191/435 amino acids; Supplementary Fig. 1b). The 
four other mammalian genes with SAC domains (SYNJ1, SYNJ2, INPP5F and SAC1) differ from Fig4 at other domains ${ }^{4}$, indicating that mouse A530089I17Rik and human KIAA0274 are homologues of yeast Fig4.

Yeast Fig4 is localized to the vacuolar membrane and is required for both generation and turnover of PtdIns $(3,5) \mathrm{P}_{2}$ (ref. 5). Yeast Fig4 exhibits lipid phosphatase activity towards the 5-phosphate residue of PtdIns $(3,5) \mathrm{P}_{2}$ (ref. 6), and also appears to activate the Fab1 kinase that synthesizes PtdIns $(3,5) \mathrm{P}_{2}$ from phosphatidylinositol-3phosphate, possibly by dephosphorylating the kinase or one of its regulators ${ }^{5}$. As a result, deletion of yeast Fig4 reduces rather than increases the intracellular concentration of $\operatorname{PtdIns}(3,5) \mathrm{P}_{2}$ (ref. 7),
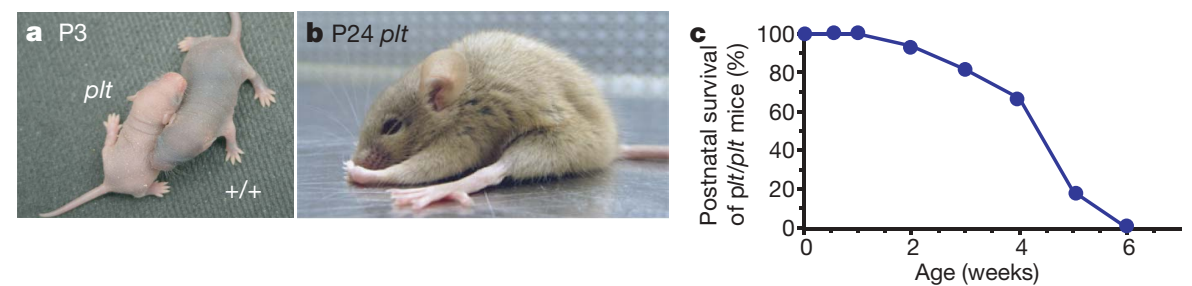

d

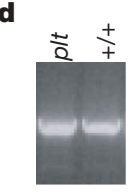

$1-8$

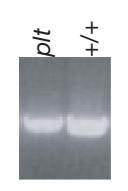

$8-15$
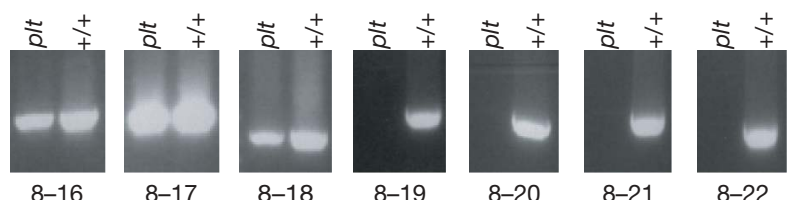

8-22

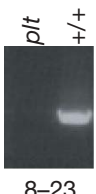

e

$\stackrel{+}{+} \frac{1}{2}$
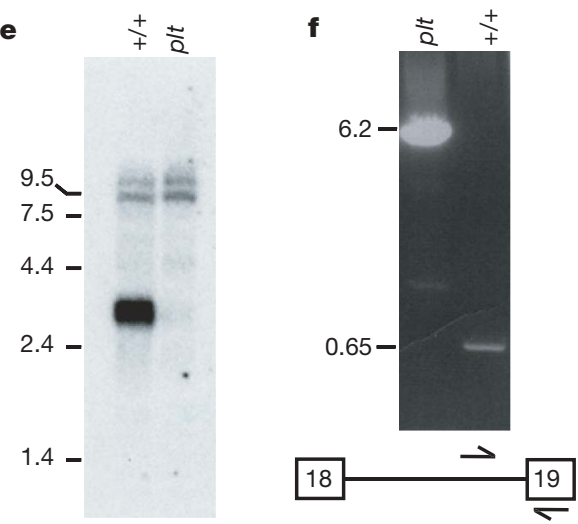

g

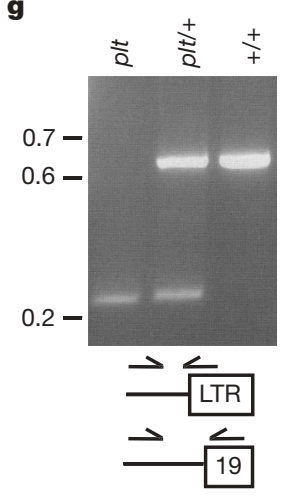

h

\begin{tabular}{lll} 
LTR & $\longrightarrow$ \\
\hline LTn2b & LTR
\end{tabular}

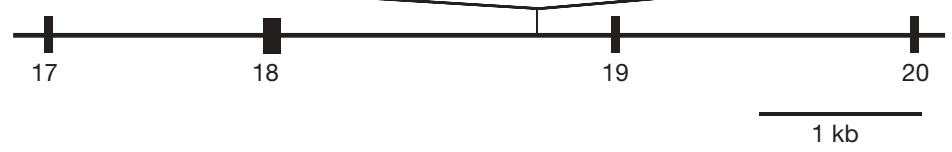

i

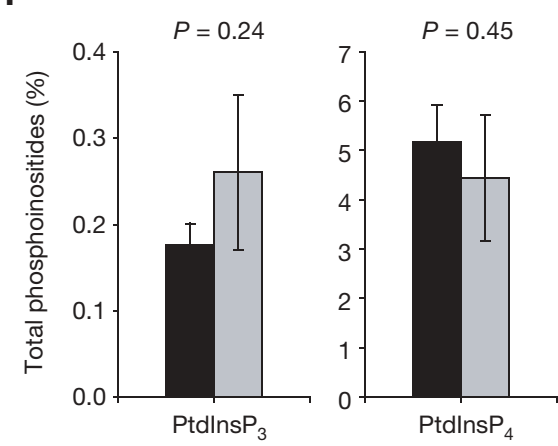

Figure 1 | Phenotypes of homozygous pale tremor mice. a, Diluted pigmentation of the pale tremor mouse (plt) compared with the wild-type $(+/+)$ mouse. $\mathbf{b}$, Abnormal limb postures. c, Juvenile lethality of $F_{2}$ mice from the CAST/Ei cross $(n=50)$. d, RT-PCR of Fig 4 transcript from brain with primers in indicated exons. e, Northern blot containing $3 \mu \mathrm{g}$ of brain polyA $^{+}$RNA, isolated at $\mathrm{P} 7$ before extensive neurodegeneration, using a $1 \mathrm{~kb}$ Fig4 cDNA probe (exons 8-15). RNA integrity and equal loading of samples
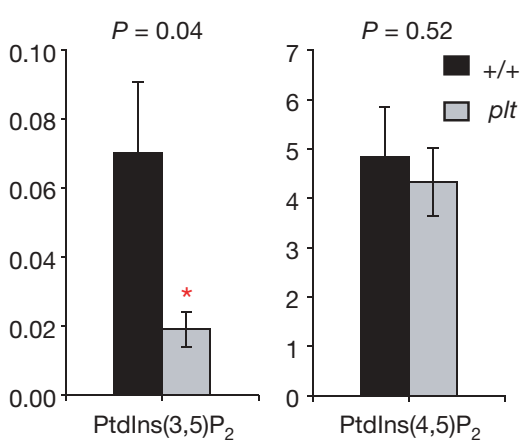

is indicated by the intensity of minor bands (9-10 kb). f, Long-range PCR of genomic DNA with primers in intron 18 and exon 19. g, Three-primer genotyping assay for Fig 4 produces $646 \mathrm{bp}$ wild-type and $245 \mathrm{bp}$ Fig $4^{\text {paletremor }}$ products. LTR, long terminal repeat. Molecular weight markers in $\mathbf{e}-\mathbf{g}$ are given in kb. h, ETn $2 \beta$ retrotransposon in intron 18 of Fig4. i, Altered abundance of PtdIns $(3,5) \mathrm{P}_{2}$ in cultured fibroblasts. ${ }^{\star}$ Significant difference at $P<0.05$. Data are expressed as mean \pm s.d. $(n=6)$. 
leading to defects in vacuole fission, formation of enlarged vacuoles and impaired retrograde traffic to the late endosome $e^{2,8,9}$. Knockdown of mammalian Fab1/PIKfyve kinase causes a similar defect in retrograde endosome traffic and enlarged vacuoles ${ }^{10}$.

Analysis of phosphoinositides from cultured fibroblasts of pale tremor mice demonstrated a three-fold reduction in $\operatorname{PtdIns}(3,5) \mathrm{P}_{2}$ $(P=0.04)$, with no change in three other phosphoinositides (Fig. 1i). Enlarged cytoplasmic vacuoles accumulate in $40 \%$ of cultured fibroblasts from pale tremor mice (174/435) compared with $5 \%$ of wild-type cells (22/403) (Supplementary Fig. 3a-d). These vacuoles stain positively for LAMP-2 (Supplementary Fig. 3e-g), indicating that they represent late-stage endosomes. The altered levels of $\operatorname{PtdIns}(3,5) \mathrm{P}_{2}$ demonstrate conserved enzymatic function of FIG4 from yeast to mouse, whereas the enlarged vacuoles demonstrate a conserved cellular role in regulation of the size of late endosomes.

In vivo loss of FIG4 results in a striking pattern of selective neurodegeneration. Extensive loss of neurons from sensory and autonomic ganglia is evident during the neonatal period (Fig. 2a-d and Supplementary Fig. 4a). The presence of neurons with enlarged cytoplasmic vacuoles suggests that vacuole accumulation precedes cell loss (Fig. 2, insets). Spinal motor neurons exhibit normal morphology at three
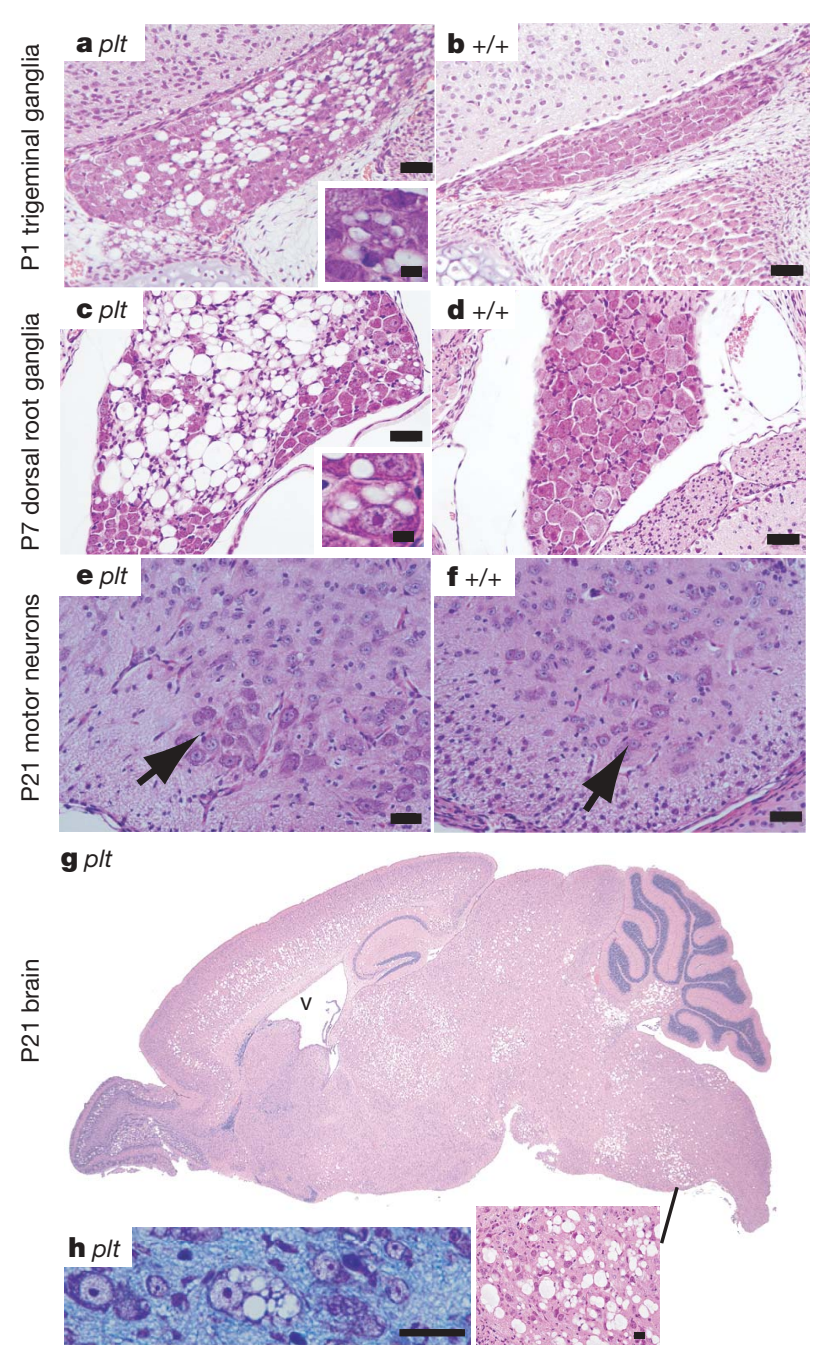

Figure 2 | Neuropathology in pale tremor mice. a, b, Trigeminal ganglia; c, d, dorsal root ganglia from lumbar region. Insets reveal cytoplasmic vacuoles. (Superior cervical ganglia have a similar appearance; see Supplementary Fig. 4a.) e, f, Spinal cord ventral horn. Arrow, motor neuron cell body. $\mathbf{g}$, Sagittal section of the brain of a pale tremor mouse (for wildtype control lacking degeneration see Supplementary Fig. 6). V, enlarged ventricle. $\boldsymbol{h}$, Neuronal cell bodies from regions of degeneration in P7 brain. Scale bars: a-f, $25 \mu \mathrm{m}$; insets in a and c, $12.5 \mu \mathrm{m}$; panel h, $25 \mu \mathrm{m}$. weeks (Fig. 2e-f) but contain vacuoles at six weeks of age (Supplementary Fig. 5).

Peripheral nerves are also affected. Cross-sections of the sciatic nerve reveal substantial reduction in the number of large-diameter myelinated axons in the mutant (Fig. 3a, b). Nerve conduction velocity and amplitude of compound muscle action potentials are reduced (Fig. 3c, d), consistent with the axonal loss visible in the sections of the sciatic nerve and the pathological abnormalities in the motor neurons. There was no response when recording from tail sensory fibres, consistent with the severe loss of sensory neurons from the dorsal root ganglia shown in Fig. 2a.

In the brain, neuronal loss in the thalamus, pons, medulla and deep cerebellar nuclei is visible at one week of age (Supplementary Fig. 6). By three weeks of age there is additional loss of neurons from layers four and five of the cortex, the deep layers of the superior and inferior colliculus and the olfactory bulb (Fig. $2 \mathrm{~g}$ ). Some neuronal cell bodies contain enlarged vacuoles (Fig. $2 \mathrm{~h}$ ); these vacuoles are not stained by Oil Red O (for lipid) or periodic acid Schiff (for carbohydrate) (not shown). Hippocampus, cerebellar cortex and cerebral cortex layers one, two, three and six remain relatively unaffected throughout the course of the disease, although under culture conditions hippocampal neurons become vacuolated (Supplementary Fig. 4b, c). The abnormal gait and motor coordination in the pale tremor mutant may be accounted for by abnormal proprioception owing to degeneration of dorsal root ganglia neurons, in combination with degeneration of neurons from brain regions directly involved in motor control (layer five of the cortex, thalamus and deep cerebellar nuclei).

Abnormalities are visible in skin and spleen. Pigment-containing hair follicles are greatly reduced in number and the few pigmented hairs contain clumped melanosomes (Supplementary Fig. 7), similar to mouse mutants with defects in lysosome-melanosome biogenesis $^{11}$. There is extensive cell loss in the spleen (Supplementary Fig. 8). White blood cell profiles are normal, and liver, kidney and testis appear normal when observed by light microscopy.

The clinical and pathological features of peripheral neuropathy in pale tremor mice resemble some types of Charcot-Marie-Tooth (CMT) disorder ${ }^{12,13}$. We tested FIG4 as a candidate gene by screening 95 individuals diagnosed with CMT disorder but lacking mutations in known genes ${ }^{13}$. The 23 coding exons of FIG4 were amplified from genomic DNA, screened by heteroduplex analysis and sequenced. Patient BAB1079 has a severe, early onset disorder. We identified the protein truncation mutation F98fsX102 in exon 4 and the missense mutation I41T in exon 2 of FIG4 (Fig. 4a). F98fsX102 truncates
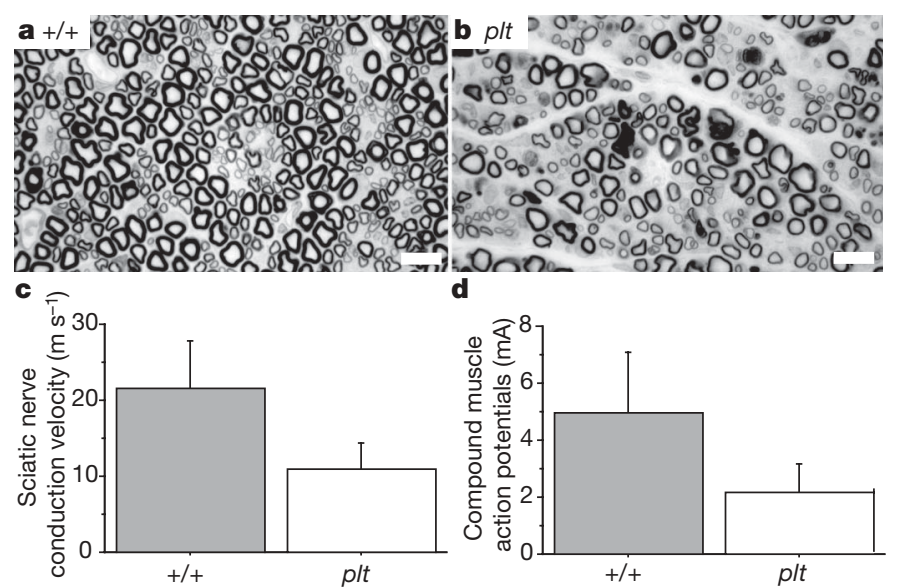

Figure 3 | Pathological abnormalities in peripheral nerves. a, b, Semi-thin sections of sciatic nerve prepared as described ${ }^{27}$, demonstrating reduced density of large-diameter myelinated axons in the pale tremor mutant. Scale bars, $10 \mu \mathrm{m}$. c, d, Reduced sciatic nerve conduction velocity

(mutant, $11.0 \pm 3.4 \mathrm{~m} \mathrm{~s}^{-1}$; wild type, $21.5 \pm 6.3 \mathrm{~m} \mathrm{~s}^{-1}$ ) and reduced amplitude of compound muscle action potentials (mutant, $2.2 \pm 1.0 \mathrm{~mA}$; wild type, $5.0 \pm 2.1 \mathrm{~mA}$ ) (mean \pm s.d., $n=6)$. 
the protein within the SAC domain and is likely to be a loss-offunction allele. Pedigree analysis demonstrated autosomal recessive inheritance. Each mutation was inherited from a heterozygous parent (Fig. 4b). Two heterozygous carriers of F98fsX102 are unaffected, indicating that FIG4 is not haploinsufficient. Heterozygous pale tremor mice at 18 months of age are also unaffected.

Another patient, BAB1372, was found to be a compound heterozygote with a nonsense mutation R183X in exon 6 together with I41T (Fig. 4a). R183X was inherited from the patient's father (Fig. 4b). The mother is an obligate carrier of I41T, and the patient has an affected sibling (BAB1373) that also inherited both mutations (Fig. 4b). Both siblings have severe disease: $\mathrm{BAB} 1372$ is functionally quadriplegic and BAB1373 is wheelchair-bound although retaining normal use of his arms. Both have slow nerve conduction velocities. A sural nerve biopsy for BAB1373 demonstrated profound axonal loss, thinly myelinated nerve fibres and evidence of de- and re-myelination.

Two additional patients, BAB1161 and BAB1369, carry unique truncation mutations together with I41T (Fig. 4a). Both patients developed disease by the age of five and demonstrate reduced nerve conduction velocity (2-7 $\mathrm{m} \mathrm{s}^{-1}$, compared with normal values of 40 $50 \mathrm{~m} \mathrm{~s}^{-1}$ ). One patient had motor developmental delay consistent with Dejerine-Sottas neuropathy.

It is remarkable that four unrelated Caucasian patients carry the same missense mutation. No additional coding or splice site variants were detected in these patients when all 23 exons of FIG4 were sequenced. Isoleucine 41 is located upstream of the SAC phosphatase domain and is evolutionarily invariant in FIG4 from yeast, invertebrates and vertebrates (Fig. 4c, d). We sequenced exon 2 from 295 neurologically normal Caucasian controls but did not identify carriers of this variant. The observed allele frequencies were 0/590 in controls and 4/190 in CMT patients $(P=0.003)$. The four patients carry I41T on the same $15 \mathrm{~kb}$ haplotype, defined by single nucleotide polymorphisms rs3799845 (G), rs2025249 (C) and rs7764711 (G) (haplotype frequency 0.29; disequilibrium coefficient, $\left.D^{\prime}=1\right)$, consistent with inheritance of a common ancestral mutant allele (see data in Supplementary Fig. 9). The evidence suggests that I41T is a rare allele causing partial loss of function that is pathogenic in combination with a null allele of FIG4.

a
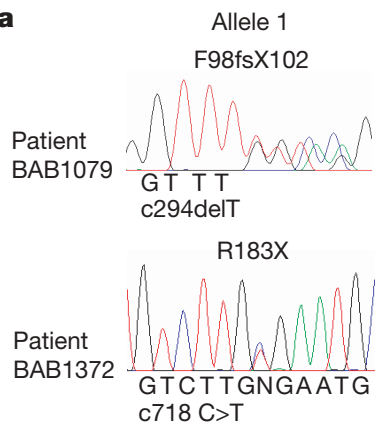
c718 C>T
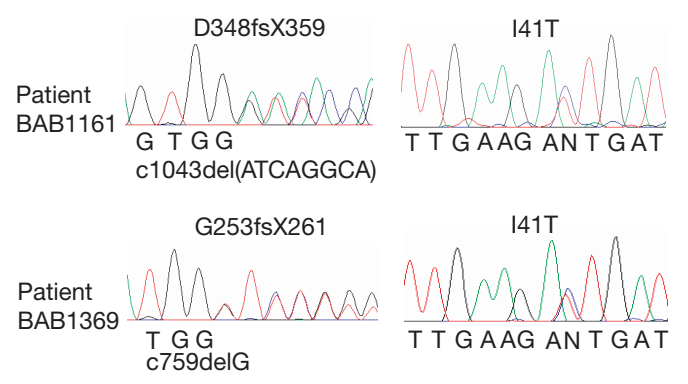

Allele 2 $141 \mathrm{~T}$

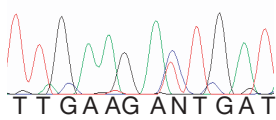
c122 $\mathrm{T}>\mathrm{C}$

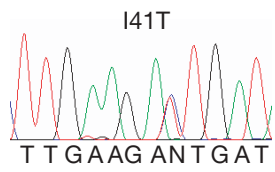

T T GAAG ANTGAT

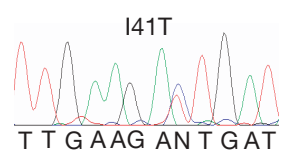

T T GAAG AN T GAT
To assess the functional effect of the I41T allele, we tested the corresponding mutation (I59T) in yeast. Wild-type and mutant cDNAs were transformed into the yeast strain fig $4 \Delta$ lacking functional Fig4 (ref.7). Transformation with the empty vector did not correct the enlarged vacuoles in fig $4 \Delta$, which reflects the slightly reduced levels of PtdIns $(3,5) \mathrm{P}_{2}$ that result from the absence of Fig4 (ref. 7) (Fig. 5a). The vacuolar enlargement was corrected to a comparable extent by wild-type and mutant Fig4, indicating that, under basal conditions, cells expressing Fig $4^{\mathrm{IS9T}}$ produce normal levels of PtdIns $(3,5) \mathrm{P}_{2}$ (Fig. 5a). The ability of the mutant to activate Fab1/PIKfyve kinase was tested by treatment with hyperosmotic shock as previously described ${ }^{5,7}$. In cells expressing wild-type Fig4, hyperosmotic shock produces a transient tenfold increase in intracellular PtdIns $(3,5) \mathrm{P}_{2}$ concentration owing to activation of Fab1/ PIKfyve kinase (Fig. 5b). In cells expressing the mutant, a partial fourfold increase was observed, demonstrating impaired activation of Fab1/PIKfyve kinase. It is not clear whether the phosphatase activity of the mutant is also impaired, owing to the low levels of $\operatorname{PtdIns}(3,5) \mathrm{P}_{2}$.

The data presented here demonstrate that mutation of FIG4 is responsible for peripheral neuronopathy in human patients. We propose the designation CMT4J for this disorder, based on the recessive inheritance pattern. Phosphoinositide signalling has previously been implicated in CMT disorder types 4B1, 4B2 and 4H (ref. 14-19) (see Supplementary Discussion). Other genes that function in vesicle trafficking, such as RAB7A and DNM2 in human ${ }^{20,21}$ and Vps54 in mouse ${ }^{22}$, are associated with inherited neuropathies.

The pale tremor mutant provides the first evidence regarding the functional role of mammalian Fig4. The results demonstrate a conserved biochemical function in metabolism of $\operatorname{Ptd} \operatorname{Ins}(3,5) \mathrm{P}_{2}$, a conserved cellular role in regulation of endosomal vesicles, and an unexpected role in neuronal survival. The molecular basis for the differential sensitivity of neuronal subtypes to loss of this widely expressed gene is unclear. Neuronal dependence on FIG4 may be related to the role of endosomal vesicles in delivering membrane components to dendritic spines during long-term potentiation ${ }^{23}$. The pale tremor mouse will generate insights into $\operatorname{Ptd} \operatorname{Ins}(3,5) \mathrm{P}_{2}$ signalling in
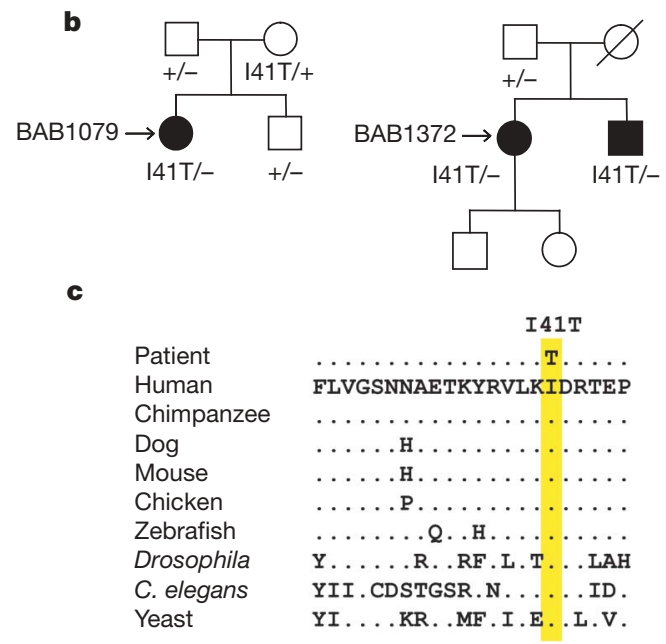

d

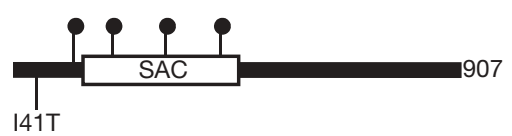

Figure 4 | Mutations of FIG4 in patients with CMT disorder. a, Sequencing chromatographs for four unrelated patients with CMT disorder. Nucleotide mutations are indicated on the $x$-axis of each chromatograph numbered from +1 for the first codon. $b$, Inheritance of mutant alleles in two pedigrees. Circle, female; square, male; open symbol, unaffected; filled symbol, affected. Patient BAB1079 is a compound heterozygote for protein truncation mutation F98fsX102 and missense mutation I41T. Patients BAB1372 and BAB1373 are compound heterozygotes for nonsense mutation R183X and missense mutation I41T. c, Residue isoleucine 41 is evolutionarily invariant in FIG4 from vertebrates, invertebrates and yeast. d, Location of CMT mutations in the FIG4 protein. Solid circles, protein truncation mutations. 907 amino acids are present per protein. 


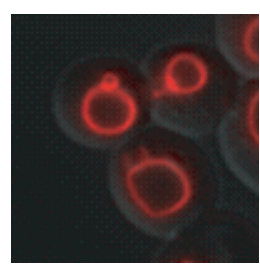

Vector

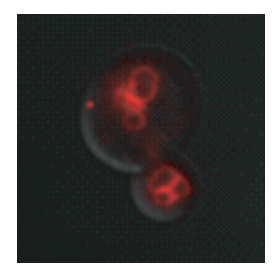

Wild type

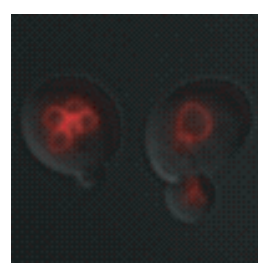

Ile $>$ Thr

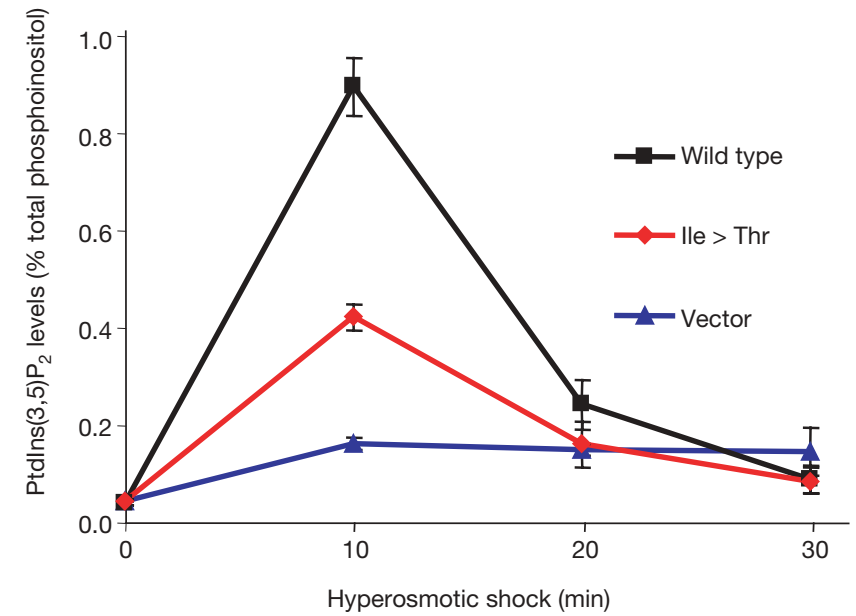

Figure 5 Yeast Fig4 $4^{\text {lle }>\text { Thr }}$ is defective in activation of kinase Fab1/ PIKfyve. The fig4 $\Delta$ yeast strain lacking endogenous Fig4 (refs 5, 7) was transformed with empty vector (vector), wild-type myc-Fig4 or myc-Fig4 containing I59T corresponding to human I41T (mutant). a, Yeast were labelled with FM4-64 to assess vacuole volume, an indicator of basal levels of PtdIns $(3,5) \mathrm{P}_{2} . \mathbf{b}$, Time course of $\operatorname{PtdIns}(3,5) \mathrm{P}_{2}$ levels after hyperosmotic shock (measured in minutes after change to hyperosmotic medium) reflects activation of Fab1 kinase. The mutant protein exhibits impaired activation at 10 min compared with the wild type $(P=0.004$; mean \pm s.d., $n=3)$. A western blot demonstrating comparable expression of wild-type and mutant constructs is presented in Supplementary Fig. 10.

neurons and provide an animal model for CMT4J and related human neuropathies.

\section{METHODS SUMMARY}

Mutation of the pale tremor gene arose on a mixed background derived from inbred mouse strains 129/Ola, C57BL/6J, C3H and SJL (ref. 24). The mutant allele is designated Fig4 paletremor . Total RNA was isolated from brain of P7 mice before extensive neurodegeneration. Primary fibroblasts were cultured from mouse tail biopsies treated with collagenase. Phosphoinositides were labelled for $24 \mathrm{~h}$ with myo- $\left[2-{ }^{3} \mathrm{H}\right]$ inositol, extracted and quantified by high-performance liquid chromatography (HPLC, see Methods). The cohort of unrelated patients with CMT disorder was previously described ${ }^{13}$. The 23 coding exons of FIG4 were screened by heteroduplex analysis ${ }^{25}$ and products with abnormal mobility were sequenced. For the four individuals with identified mutations, all of the other exons were sequenced. Genomic DNA from neurological normal control individuals was obtained from the Coriell Institute (NDPT006 and NDPT009, 96 samples each) and from a collection of 111 subjects older than 60 without personal or family history of neurological disease ${ }^{26}$.

Full Methods and any associated references are available in the online version of the paper at www.nature.com/nature.

\section{Received 7 March; accepted 23 April 2007}

Published online 17 June 2007.

1. Di Paolo, G. \& De Camilli, P. Phosphoinositides in cell regulation and membrane dynamics. Nature 443, 651-657 (2006).

2. Michell, R. H., Heath, V. L., Lemmon, M. A. \& Dove, S. K. Phosphatidylinositol 3,5bisphosphate: metabolism and cellular functions. Trends Biochem. Sci. 31, 52-63 (2006).

3. Maksakova, I. A. et al. Retroviral elements and their hosts: insertional mutagenesis in the mouse germ line. PLoS Genet. 2, e2 (2006).
4. Hughes, W. E., Cooke, F. T. \& Parker, P. J. Sac phosphatase domain proteins. Biochem. J. 350, 337-352 (2000).

5. Duex, J. E., Tang, F. \& Weisman, L. S. The Vac14p-Fig4p complex acts independently of Vac7p and couples PI3,5 $\mathrm{P}_{2}$ synthesis and turnover. J. Cell Biol. 172, 693-704 (2006).

6. Rudge, S. A., Anderson, D. M. \& Emr, S. D. Vacuole size control: regulation of Ptdlns $(3,5) \mathrm{P}_{2}$ levels by the vacuole-associated Vac14-Fig4 complex, a Ptdlns(3,5) $\mathrm{P}_{2}$-specific phosphatase. Mol. Biol. Cell 15, 24-36 (2004).

7. Duex, J. E., Nau, J. J., Kauffman, E. J. \& Weisman, L. S. Phosphoinositide 5 -phosphatase Fig $4 p$ is required for both acute rise and subsequent fall in stressinduced phosphatidylinositol 3,5-bisphosphate levels. Eukaryot. Cell 5, 723-731 (2006).

8. Bonangelino, C. J. et al. Osmotic stress-induced increase of phosphatidylinositol 3,5-bisphosphate requires Vac14p, an activator of the lipid kinase Fab1p. J. Cell Biol. 156, 1015-1028 (2002).

9. Gary, J. D. et al. Regulation of Fab1 phosphatidylinositol 3-phosphate 5-kinase pathway by Vac7 protein and Fig4, a polyphosphoinositide phosphatase family member. Mol. Biol. Cell 13, 1238-1251 (2002).

10. Rutherford, A. C. et al. The mammalian phosphatidylinositol 3-phosphate 5-kinase (PIKfyve) regulates endosome-to-TGN retrograde transport. J. Cell Sci. 119, 3944-3957 (2006).

11. Marks, M. S. \& Seabra, M. C. The melanosome: membrane dynamics in black and white. Nature Rev. Mol. Cell Biol. 2, 738-748 (2001).

12. Schroder, J. M. Neuropathology of Charcot-Marie-Tooth and related disorders. Neuromolecular Med. 8, 23-42 (2006).

13. Szigeti, K., Garcia, C. A. \& Lupski, J. R. Charcot-Marie-Tooth disease and related hereditary polyneuropathies: molecular diagnostics determine aspects of medical management. Genet. Med. 8, 86-92 (2006).

14. Begley, M. J. et al. Molecular basis for substrate recognition by MTMR2, a myotubularin family phosphoinositide phosphatase. Proc. Natl Acad. Sci. USA 103, 927-932 (2006).

15. Bolino, A. et al. Disruption of Mtmr2 produces CMT4B1-like neuropathy with myelin outfolding and impaired spermatogenesis. J. Cell Biol. 167, 711-721 (2004).

16. Bolino, A. et al. Charcot-Marie-Tooth type $4 \mathrm{~B}$ is caused by mutations in the gene encoding myotubularin-related protein-2. Nature Genet. 25, 17-19 (2000).

17. Bonneick, S. et al. An animal model for Charcot-Marie-Tooth disease type 4B1. Hum. Mol. Genet. 14, 3685-3695 (2005).

18. Senderek, J. et al. Mutation of the SBF2 gene, encoding a novel member of the myotubularin family, in Charcot-Marie-Tooth neuropathy type 4B2/11p15. Hum. Mol. Genet. 12, 349-356 (2003)

19. Stendel, C. et al. Peripheral nerve demyelination caused by a mutant Rho GTPase guanine nucleotide exchange factor, frabin/FGD4. Am. J. Hum. Genet. (in the press); preprint at http://www.journals.uchicago.edu/AJHG/journal/preprints/ AJHG44688.preprint.pdf (2007).

20. Verhoeven, K. et al. Mutations in the small GTP-ase late endosomal protein RAB7 cause Charcot-Marie-Tooth type 2B neuropathy. Am. J. Hum. Genet. 72, 722-727 (2003).

21. Zuchner, S. et al. Mutations in the pleckstrin homology domain of dynamin 2 cause dominant intermediate Charcot-Marie-Tooth disease. Nature Genet. 37, 289-294 (2005)

22. Schmitt-John, T. et al. Mutation of Vps54 causes motor neuron disease and defective spermiogenesis in the wobbler mouse. Nature Genet. 37, 1213-1215 (2005).

23. Park, M. et al. Plasticity-induced growth of dendritic spines by exocytic trafficking from recycling endosomes. Neuron 52, 817-830 (2006)

24. Adamska, M., Billi, A. C., Cheek, S. \& Meisler, M. H. Genetic interaction between Wnt7a and Lrp6 during patterning of dorsal and posterior structures of the mouse limb. Dev. Dyn. 233, 368-372 (2005).

25. Escayg, A. et al. Mutations of SCN1A, encoding a neuronal sodium channel, in two families with GEFS + 2. Nature Genet. 24, 343-345 (2000).

26. Rainier, S., Sher, C. Reish, O., Thomas, D. \& Fink, J. K. De novo occurrence of novel SPG3A/atlastin mutation presenting as cerebral palsy. Arch. Neurol. 63, 445-447 (2006).

27. Li, J. et al. Major myelin protein gene (PO) mutation causes a novel form of axonal degeneration. J. Comp. Neurol. 498, 252-265 (2006).

28. Kohrman, D. C. Harris, J. B. \& Meisler, M. H. Mutation detection in the med and med alleles of the sodium channel Scn8a. Unusual splicing due to a minor class AT-AC intron. J. Biol. Chem. 271, 17576-17581 (1996).

Supplementary Information is linked to the online version of the paper at www.nature.com/nature.

Acknowledgements For discussions and advice we are grateful to A. Dlugosz, E. Feldman, D. Goldowitz, J. Hammond, L. Isom, J. M. Jones, A. Lieberman, M. Khajavi, J. Swanson, K. Verhey and S. H. Yang. S. Cheek and M. Hancock provided technical assistance. This research was supported by NIH research grants (M.H.M., L.W. and J.R.L.) and NIH predoctoral training (C.Y.C.).

Author Information Reprints and permissions information is available at www.nature.com/reprints. The authors declare no competing financial interests. Correspondence and requests for materials should be addressed to M.H.M. (meislerm@umich.edu). 


\section{METHODS}

Animals. For genetic mapping, plt/ + heterozygotes were crossed with strain CAST/Ei (Jackson Laboratory). Experiments were carried out on $\mathrm{F}_{2}$ and $\mathrm{F}_{3}$ mice from the mapping cross. This research was approved by the University of Michigan Committee on Use and Care of Animals. Animals were housed and cared for in accordance with NIH guidelines.

Genotyping and markers. Genotyping was carried out using microsatellite markers from public databases as well as novel microsatellite markers designed from mouse genomic sequence (http://www.ensemble.org). D10Umil3 was amplified with the forward primer $5^{\prime}$-CCACCACATCAACAGGCTCACAGG and the reverse primer 5'-AATGCAACCGTGACACAAGTACAC. PCR was carried out with the PCR core kit (Qiagen). PCR products were separated on 6\% acrylamide gels and stained with ethidium bromide. The pale tremor mutation is genotyped by PCR with a forward primer in intron 18 (5'-CGTATGAATTGAGTAGTTTTGATG) and two reverse primers, one in the proximal LTR of the inserted $E T n 2 \beta$ element (5'-GCTGGGGGAGGGGAGACTACACAG) and one in exon 19 (5'-ATGGACTTGGATCAATGCCAACAG).

RT-PCR. Total RNA was isolated from brain of P7 mice, before extensive neurodegeneration. cDNA was synthesized using the First Strand cDNA Synthesis Kit (Invitrogen). RT-PCR was carried out with the PCR Core Kit (Qiagen). Long-range PCR was performed with the Expand Long Template PCR System (Roche). The northern blot with $3 \mu \mathrm{g}$ of polyA ${ }^{+}$RNA was prepared as previously described $^{28}$. The hybridization probe, a $1 \mathrm{~kb}$ RT-PCR product containing exons 8 to 15 , was labelled with two radiolabelled nucleotide triphosphates.

Histology. Tissues were sectioned and stained at HistoServ. Fast blue/eosin staining was carried out in the Department of Pathology, University of Michigan. Light microscopy was performed on an Olympus BX-51 microscope and DP50 camera. Sciatic and femoral nerves were sectioned and stained with osmium for electron microscopy as previously described ${ }^{27}$. Skin whole mounts were prepared from P10 mice with the guidance of A. Dlugosz. The commercial depilatory Nair was applied to the dorsal surface for five minutes followed by washing with warm water to remove hair. The skin was dissected and superficial fascia removed. Follicles were visualized on a standard dissecting microscope with transmitted light.

Neurophysiology. Nerve conduction velocities were recorded from affected pale tremor mice and littermate controls. Mice were anaesthetized with a ketamine/ xylazine solution and placed under a heating lamp to maintain body temperature at $32^{\circ} \mathrm{C}$. Recordings were obtained using a Nicolet VikingQuest portable system and Nicolet disposable EEG needles. Tail sensory responses were obtained by stimulating proximally over a $3 \mathrm{~cm}$ region. Sciatic nerve motor velocities were obtained by stimulating distally at the sciatic notch and proximally at the knee. Cell culture and immunofluorescence. Primary fibroblasts were cultured from mouse tail biopsies treated with collagenase. Cells were plated in DMEM with $10 \%$ fetal bovine serum, and maintained at $37^{\circ} \mathrm{C}$ with $5 \% \mathrm{CO}_{2}$ for up to three passages. For immunofluorescence, 100,000 cells were seeded on polylysinecoated cover slips in $35 \mathrm{~mm}$ dishes. We thank T. August and the Developmental Studies Hybridoma Bank for monoclonal antibody to LAMP-2, developed under the auspices of the NICHD and maintained by the Department of Biological Sciences, University of Iowa. For labelling with antibody, cells were fixed with ice-cold methanol at $-20^{\circ} \mathrm{C}$ for $5 \mathrm{~min}$ and blocked with $2 \%$ goat serum. Antibodies were applied for $1 \mathrm{~h}$ in PBS with 2\% serum at room temperature and detected with Alexa Fluor 488 donkey anti-rabbit (Molecular Probes) or Alexa Fluor 594 goat anti-rat (Molecular Probes). Cells were visualized on a DeltaVision Deconvolution microscope system (Applied Precision).

Hippocampal neurons were cultured with glial conditioned media. Neurons were visualized with a Nikon TE2000 microscope.

Phosphoinositide assays. Fibroblast phosphoinositides were labelled with myo$\left[2-{ }^{3} \mathrm{H}\right]$ inositol, extracted and quantified by HPLC. Mouse fibroblasts from the first passage were grown in $100 \mathrm{~mm}$ dishes to $60-70 \%$ confluency. The culture was rinsed with PBS and starved for $12 \mathrm{~h}$ in inositol-free DMEM (Tissue Culture Support Centre, Washington University) supplemented with $5 \mu \mathrm{g} \mathrm{ml}^{-1}$ transferrin, $5 \mu \mathrm{g} \mathrm{ml}^{-1}$ insulin and $10 \%$ dialysed fetal bovine serum. The medium was replaced with labelling medium (inositol-free DMEM containing $5 \mu \mathrm{g} \mathrm{ml}^{-1}$ transferrin, $20 \mathrm{mM}$ HEPES and $50 \mu \mathrm{Ci}$ myo- $\left[2-{ }^{3} \mathrm{H}\right]$ inositol, GE Healthcare). After $24 \mathrm{~h}$, the culture was treated with $0.6 \mathrm{ml}$ of $4.5 \%(\mathrm{v} / \mathrm{v})$ perchloric acid for $15 \mathrm{~min}$, scraped off the plate, and spun down at $12,000 \mathrm{~g}$ for $10 \mathrm{~min}$ at $4{ }^{\circ} \mathrm{C}$. The pellet was washed with $0.1 \mathrm{M}$ EDTA once and resuspended in $50 \mu \mathrm{l}$ deionized water. To deacylate the lipids, samples were transferred to a glass vial, mixed with $1 \mathrm{ml}$ methanol $/ 40 \%$ methylamine $/ n$-butanol $(4: 4: 1, \mathrm{v} / \mathrm{v})$, and incubated at $55^{\circ} \mathrm{C}$ for $1 \mathrm{~h}$. The resulting samples were vacuum-dried, resuspended in $0.3 \mathrm{ml}$ water and extracted twice with an equal volume of $n$-butanol/ethyl ether/formate (20:4:1, v/v). The aqueous phase was vacuum dried and resuspended in $20 \mu \mathrm{l}$ water.
For separation of all isoforms of the glycerophosphoinositides by HPLC, two different elution gradients were used at $1 \mathrm{ml} \mathrm{min}^{-1}$ flow rate. (Pump A, $\mathrm{H}_{2} \mathrm{O}$; pump B, $1 \mathrm{M}\left(\mathrm{NH}_{4}\right)_{2} \mathrm{HPO}_{4}, \mathrm{pH} 3.8$.) Gradient 1: $0 \%$ B for $5 \mathrm{~min} ; 0-2 \%$ B over $15 \mathrm{~min}$; $2 \%$ B for 80 min; $2-12 \%$ B over $20 \mathrm{~min} ; 12 \%$ B for 20 min; $12-80 \%$ B over 40 min; $80 \%$ B for $20 \mathrm{~min} ; 80-0 \%$ B over $5 \mathrm{~min}$. To separate glyceroPtdIns $(3,4) \mathrm{P}_{2}$ from glyceroPtdIns $(3,5) \mathrm{P}_{2}$, a longer gradient (gradient 2) was used: $0 \% \mathrm{~B}$ for $5 \mathrm{~min}$; $0-2 \%$ B over $15 \mathrm{~min} ; 2 \%$ B for $80 \mathrm{~min} ; 2-10 \%$ B over $20 \mathrm{~min} ; 10 \%$ B for $65 \mathrm{~min} ; 10$ $80 \%$ B over $40 \mathrm{~min} ; 80 \%$ B for $20 \mathrm{~min} ; 80-0 \%$ B over $5 \mathrm{~min}$. The positions of glyceroPtdIns(3)P, glyceroPtdIns $(3,5) \mathrm{P}_{2}$, glyceroPtdIns $(3,4) \mathrm{P}_{2}$ and glyceroPtdIns $(3,4,5) \mathrm{P}_{3}$ were determined by ${ }^{32} \mathrm{P}$-labelled standards received as gifts from $\mathrm{L}$. Rameh. The positions of glyceroPtdIns(4)P and glyceroPtdIns $(4,5) \mathrm{P}_{2}$ were confirmed with yeast glycerophosphoinositide extracts.

Human mutation detection. The cohort of unrelated patients with CMT disorder was previously described ${ }^{13}$. The clinical diagnosis was based on clinical examination, electrophysiological studies and, in a few cases, nerve biopsy. All patients received appropriate counselling and gave informed consent approved by the institutional review board. For the initial screen of FIG4, each coding exon was amplified and examined by heteroduplex analysis as previously described ${ }^{25}$. The patient mutations were identified by sequencing products exhibiting abnormal mobility. Subsequently, the 23 exons of FIG4 were completely sequenced from the four individuals carrying variants. 